\title{
Administración de fluoruros en salud pública en el Perú. Debilidades y obstáculos
}

Fluorides management in public health in Perú. Weaknesses and obstacles

Raúl Vallejos-Ragas ${ }^{1, a}$, Pamela Tineo-Tueros ${ }^{2, b}$

\section{RESUMEN}

La eficiencia del uso de fluoruros por vía sistémica, tanto en agua o sal de consumo humano, es un tema poco discutido hoy en día, esto contrasta con la escasa información de efectividad y logros en el Perú, en la reducción de caries dental. El artículo describe las diversas experiencias de aplicación de fluoruros adoptadas en el país, tanto por vía sistémica como por aplicación tópica, donde se evidencian los diversos criterios para la elección de la forma de aplicación de fluoruros por parte de las autoridades de turno, el débil desempeño de las diversas gestiones y la falta de continuidad de los procesos. Así mismo, se describe el Análisis Costo-Efectividad (ACE) aplicado a las diversas formas de fluoruros.

PALABRAS CLAVE: Fluoruros, fluoruros tópicos, fluoruración, análisis costo-efectividad, salud pública. (DeCS, BIREME) 


\section{SUMMARY}

The efficiency of the use of fluorides for systemic, in water or salt for human consumption is a subject little discussed today, this contrasts with the limited information on the effectiveness and achievements in Peru, reducing tooth decay. The article describes the experiences of applying various fluorides taken in the country, both by systemically and topically applied, which show the various criteria for the choice of the mode of application of fluoride by the authorities, the weak performance of the various steps and lack of continuity of the processes. It also describes the Cost-Effectiveness analysis (ACE) applied to the various forms of fluoride.

\section{KEYWORDS: Fluorides, fluorides topical, fluoridation, cost-effectiveness, public health. (MeSH-NLM)}

\section{INTRODUCCIÓN}

Las múltiples evidencias científicas sobre la eficiencia de los fluoruros de aplicación sistémica (en agua o sal de consumo humano) en la prevención de la caries dental, contrastan con la escasa información de efectividad y de logros concretos en la reducción de la caries dental en el Perú. Contrariamente, las acciones más frecuentes desarrolladas en el campo de la salud pública, utilizan fluoruros de uso tópico, al parecer en la búsqueda del mismo impacto que el logrado por los fluoruros sistémicos; conllevando así, a que muchos programas, proyectos y campañas dirigidos a diversas poblaciones, incorporen estos esquemas sin considerar su costo-efectividad y la factibilidad para su implementación. Contribuyen a esto, las debilidades del estado en garantizar políticas de salud bucal, que incluyan un programa eficiente de administración de sal fluorada, así como la gran competencia de odontólogos del sector privado, que utilizan campañas de aplicación de fluoruros tópicos, como mecanismo de captación de pacientes, producto de las reglas del mercado imperantes en el país.

El presente artículo tiene como fin realizar una revisión histórica de las experiencias de aplicación de fluoruros en el Perú, describir el Análisis de CostoEfectividad (ACE) de las formas de administración de fluoruros, acompañado de la identificación de las posibles causas de la ausencia de evidencias de impacto con estas experiencias.

\section{Antecedentes del uso de fluoruros sistémicos}

Los intentos por adicionar flúor al agua de consumo en el Perú se inician a finales de los 50', con experiencias piloto en pequeñas zonas urbanas de Lima y Chimbote (provincia al norte de Lima). Intentando a partir de 1973 la aplicación masiva en la ciudad de Lima, la que tuvo una duración de escasos cinco meses, después de lo cual se abandonaron los intentos por problemas técnicos en la principal planta de tratamiento de agua de la ciudad (La Atarjea) (1).

Al cabo de diez años, el país adopta la aplicación de flúor sistémico a través de la sal de consumo humano, como parte del impulso de este modelo en la región. La obligatoriedad de su aplicación a nivel nacional se estableció en 1984 (2) y un año después se publicaron las normas para la adición (3). Posteriormente se crea el Programa Nacional de Fluoruración de la Sal, el cual recibe la donación de $\$ 495000$ para dicho fin, por parte de la fundación E K Kellogg. Programas similares fueron desarrollados en esa época en países como Costa Rica, Jamaica y México, que recibieron financiamiento parcial por la mencionada fundación (4), los cuales lograron resultados muy favorables, como se muestra en la Tabla 1.

Tabla 1. Impacto de la sal fluorada en la reducción del COPD.

\begin{tabular}{llcccc}
\hline \multicolumn{1}{c}{ País } & \multicolumn{2}{c}{ Estudio de base } & \multicolumn{2}{c}{ Siguiente estudio } & $\begin{array}{c}\% \\
\text { Reducción } \\
\end{array}$ \\
& Año & CPOD & Año & CPOD & de caries \\
\hline Costa Rica & 1988 & 8,4 & 1996 & 4,8 & 49 \\
Jamaica & 1984 & 6,7 & 1995 & 1,1 & 83 \\
México & 1984 & 2,32 & 1999 & 1,39 & 40 \\
\hline
\end{tabular}


Entre los años 89 y 90, el programa subscribe convenios con dos empresas privadas de elaboración de sal de consumo humano. En los mismos, el Ministerio de Salud (MINSA) se comprometía a entregar equipos para la adición de flúor a la sal, con la condición de que estas empresas elaboren sal fluorada en el país $(5,6)$.

Como parte de la implementación del programa, la Organización Panamericana de la Salud (OPS), recomendó realizar dos estudios de base y dos complementarios. El primer grupo correspondía a investigaciones de a) caries dental y fluorosis, y b) niveles basales de fluoruros en la red de agua de consumo; mientras que, el segundo grupo de estudios se orientaba a c) niveles de excreción de fluoruros en orina en niños de 3 a 5 años de edad y d) evaluación de otras fuentes disponibles de fluoruros. El primer estudio epidemiológico nacional de caries dental realizado a inicios del programa, (que no incluía fluorosis) (7), presentó debilidades metodológicas; siendo la más resaltante el insuficiente número muestral para la edad 12 años. Los otros tres estudios no fueron realizados, por lo cual no se dispone de dicha información. La fundación Kellogg al no observar avances en el programa, decide no continuar con los desembolsos, habiendo cumplido hasta finales

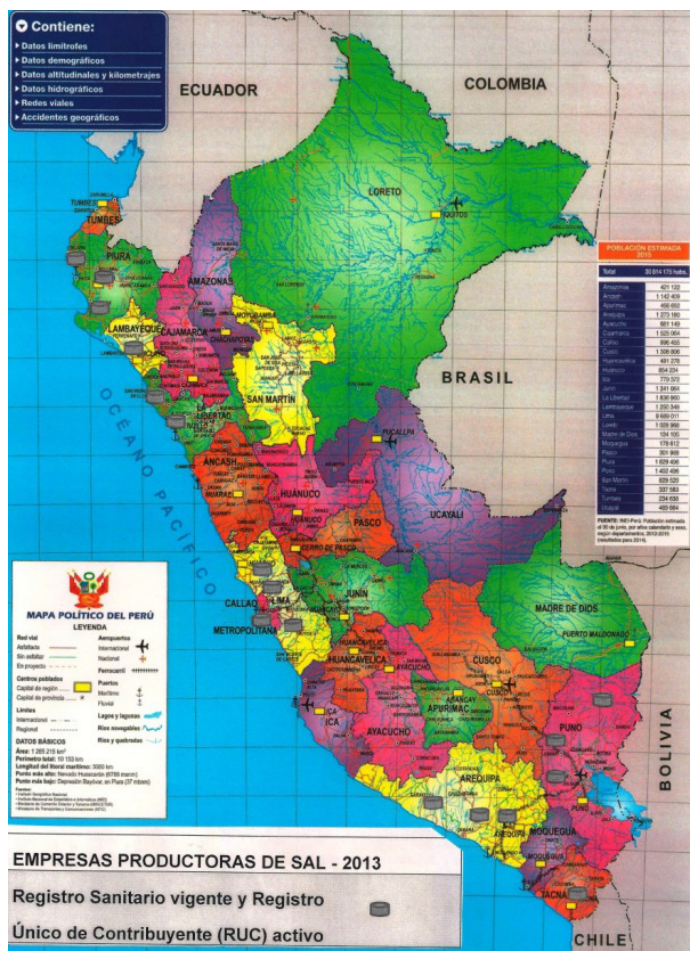

Figura 1. Empresas formales productoras de sal en el Perú, 2013. de los 90 ' con la entrega del $60 \%$ del total ofrecido (8).

Los intentos por fortalecer la fluorización de la sal de consumo, se dieron en forma irregular durante los años posteriores, donde se desconocía si las empresas productoras cumplían con la adición de fluoruro a la sal (200 ppm) (3) y si las zonas de distribución incluían localidades donde el flúor en agua superaba el máximo recomendado (1000 ppm) (9). En la última década el número de empresas se redujeron de 60 a 17 , considerando a estas últimas, las únicas que cumplen la formalidad de tener el Registro Sanitario Vigente (otorgado por el MINSA) y la Actividad Tributaria Activa (otorgado por el ente recaudador de impuestos del país - SUNAT) (Figura 1). Sin embargo, existen otras 17 empresas con Registro Sanitario Vencido, pero con Actividad Tributaria Activa $(10,11)$.

De la totalidad de empresas formales, el $82.35 \%$ se encuentran a lo largo de la costa del país. Estando las restantes ubicadas en la sierra sur del país (Puno). Siendo la de mayor volumen de producción la ubicada en Lima (12).

El proceso de implementación de un programa de fluorización de sal, ha sido clara y ampliamente detallado por la Organización Panamericana de la Salud (OPS). Sin embargo la descripción histórica de este proceso en el Perú ha evidenciado algunas debilidades del ente rector en salud (MINSA), que están por encima del efecto mismo del flúor en la prevención de la caries. Entre estos puntos se pueden identificar (13):

a) El pobre desempeño del Sistema de Salud peruano (puesto 129 de 172 países).

b) La débil institucionalidad del MINSA, reflejada en la falta de continuidad de procesos y de profesionales asignados para esta labor, $\mathrm{y}$

c) Los escasos procesos de capacitación desarrollados en el país, relacionados a Salud Pública bucal, que se reflejan en la ausencia de indicadores y en las decisiones de los equipos técnicos que dirigen la política de salud bucal peruana.

En 1999, se inició un proyecto de fluoruración de la leche, en un distrito de la ciudad de Trujillo, en la costa norte del país, financiado por la Borrow Dental Milk Fundation. El proyecto se asociaba al programa gubernamental del "vaso de leche" y estuvo dirigido a niños menores de 6 años de edad. La leche fue 
suplementada con $0,25 \mathrm{mg}$ de fluoruro y era preparada y distribuida en los "clubs de madres" del distrito. En el 2004, el proyecto fue detenido $(14,15)$.

\section{Antecedentes del uso de fluoruros tópicos}

La referencia sobre el uso de fluoruros tópicos en el Perú, data de 1964, donde se describe que dicha aplicación debe realizarse con una solución acuosa de fluoruro de sodio al $2 \%$ sobre las superficies de los dientes. Recomendando, que éstas sean realizadas en cuatro aplicaciones, en cuatro tiempos diferentes de la vida del niño (3,7,10 y 13 años de edad) (16). Un esquema muy parecido fue incorporado en esa época en actividades preventivas del Sub Programa Nacional de Salud Bucal del MINSA. Estas consistían en la aplicación de fluoruro de sodio al $2 \%$ por cuatro días consecutivos a niños en edad preescolar, repitiéndose esto cada año. Ambos esquemas preventivos en la actualidad ya no se utilizan.

Desde 1984, se reporta la aplicación de otro esquema, como parte de un programa de la Municipalidad Metropolitana de Lima, incorporando así, los bucales de flúor al $0.2 \%$. El MINSA lo implementó a partir de 1985, orientándolo a niños de escuelas públicas (17) y lo continúo hasta el año 2008. Esta aplicación estuvo a cargo de odontólogos del sector público y en algunos momentos por docentes de las escuelas. Los programas consideraban como mecanismo de control el reporte de cobertura, adoleciendo de indicadores de impacto (18).

A consecuencia de problemas en el nivel operativo de la aplicación de enjuagatorios, el Programa Nacional de Salud Bucal del MINSA, consideró reforzar las actividades preventivas mediante la aplicación de flúor fosfato acidulado al $1.23 \%$ en forma de gel. El que se implementó, mediante programas pilotos entre 1995 y el 2000. Esta actividad fue desarrollada como complemento a los enjuagatorios (19).

El año 2001, el MINSA publicó la norma que regula la adición de fluoruros a las cremas dentales y enjuagatorios (20). El uso de cremas dentales fluoradas, fue fomentado por iniciativa de las empresas productoras. A esto se sumó un gran impulso a partir de 1995, a consecuencia de un convenio suscrito entre el Colegio Odontológico del Perú (COP) y una empresa privada fabricante de cremas dentales, mediante el cual, esta última hacia entrega de 100 000 kits dentales anuales (crema y cepillo dental) y el COP se comprometía a distribuir dichos productos en todo el país. Convirtiéndose de esa forma la entrega de los kits en la principal actividad "preventiva" desarrollada por el COP, durante los siguientes 15 años $(21,22,23)$.

Tabla 2. Valores de análisis de costo-efectividad de aplicaciones de fluoruros.

\begin{tabular}{|c|c|c|c|c|}
\hline Formas de aplicación & $\begin{array}{l}\text { Costo aproximado } \\
\text { anual en \$ }\end{array}$ & Aplicaciones al año & $\begin{array}{l}\text { Efectividad aproximada } \\
\text { de reducción de caries }\end{array}$ & $\mathbf{A C E}$ \\
\hline Sal fluorada & $\$ 0,06$ por persona & $\begin{array}{l}\text { Entre } 7,8 \mathrm{gr} \text { a } 10 \mathrm{gr} \text { por } \\
\text { persona al día }\end{array}$ & $50 \%$ & 0,12 \\
\hline Agua fluorada & $\begin{array}{l}\text { Entre } \$ 0,51 \text { y } \$ 0,72 \\
\text { por persona }\end{array}$ & Consumo diario & entre 18 y $40 \%$ & 2,12 \\
\hline $\begin{array}{l}\text { Enjuagatorios con } \\
\text { solución fluorada } \\
(0.2 \%)\end{array}$ & $\begin{array}{c}\text { Entre } \$ 0,54 \text { a } \$ 2,54 \\
\text { por niño }\end{array}$ & 24 aplicaciones & $31 \%$ & 4,96 \\
\hline Leche fluorada & $\begin{array}{c}\text { Entre } \$ 2,43 \text { y } \$ 3,49 \\
\text { por niño }\end{array}$ & Consumo diario & $43 \%$ & 6,88 \\
\hline Barniz de Flúor & $\begin{array}{c}\text { Entre } \$ 4 \text { a } \$ 8 \text { por } \\
\text { niño }\end{array}$ & 2 a 4 aplicaciones & entre $20-50 \%$ & 17,14 \\
\hline Crema Dental fluorada & $\begin{array}{c}\text { Entre } \$ 6 \text { a } \$ 12 \text { por } \\
\text { persona }\end{array}$ & $\begin{array}{l}\text { Cepíllese dos veces al día } \\
\text { utilizando } 0,25 \mathrm{gr} \text { crema } \\
\text { dental ( } 2 \text { tubos al año) }\end{array}$ & $24 \%$ & 37,5 \\
\hline $\begin{array}{l}\text { Suplementos de Flúor } \\
\text { de la Dieta }\end{array}$ & $\$ 37$ por persona & & $43 \%$. & 86,04 \\
\hline Flúor en gel & $\$ 23$ & 3 a 4 aplicaciones & $26 \%$ & 88,46 \\
\hline
\end{tabular}




\section{Valores de costo efectividad de los fluoruros}

Los antecedentes sobre el uso de fluoruros en el país, muestran que los criterios adoptados para la elección de una forma de aplicación, no siempre surgían del uso de herramientas técnicas, entre ellas el Análisis de Costo-Efectividad (ACE). El mismo que se define como "el procedimiento para determinar sistemáticamente y objetivamente la relación entre los costos y beneficios de las intervenciones preventivas" (24). Esta evaluación económica se utiliza para comparar dos o más intervenciones y elegir la mejor desde el punto de vista económico y clínico. El Análisis Costo-Efectividad, expresa el beneficio obtenido en valores no monetarios, siendo más bien, un coeficiente la forma de expresión adecuada (25).

El ACE en realidad comprende tres procesos distintos (26):

- Análisis de los costos de cada alternativa, expresado en términos monetarios.

- Análisis de efectividad de cada alternativa, expresado en términos no monetarios.

- Análisis de la relación entre los costos y la efectividad de cada alternativa, coeficiente dividido entre el costo por la efectividad.

En resumen, el ACE nos permitirá establecer el logro de un determinado objetivo con el mínimo gasto de recursos. Sin embargo, se debe tener en cuenta que las alternativas a evaluar deben ser comparables. Por ejemplo, estar dirigidas a los mismos grupos de destinatarios (26).

Los valores de costo y efectividad de las diversas formas de aplicación de fluoruros son información ya descrita, pero no consolidada, siendo de gran utilidad para la elección en programas de salud pública bucal. Es importante considerar que los valores de costos y efectividad presentados en la Tabla 2, fueron obtenidos de diversas fuentes $(27,28,29)$, desconociendo las metodologías utilizadas. Así mismo, los valores de costo o efectividad no fueron constantes, lo que hubiera facilitado el análisis. Tal es el caso, de la efectividad de la leche fluorada y los suplementos de flúor en la dieta (43\%), donde se hace evidente que el costo es muy superior en el último método. Teniendo en cuenta estas consideraciones, el Análisis de Costo-Efectividad (ACE) obtenido, resulta ser muy ilustrativo $(27,28)$.

\section{REFLEXIÓN FINAL}

Las diversas experiencias desarrolladas en el ámbito de la salud publica bucal en el Perú y el escaso logro de evidencias tangibles, se convierten en la oportunidad de iniciar reflexiones, discusiones y análisis en la que podamos identificar la totalidad de factores que influyeron y vienen influyendo en el desempeño de nuestras acciones en este campo, convirtiéndose de esta forma en el punto de partida para construir un proceso técnico, sostenible y de impacto en la salud bucal de la población.

\section{Correspondencia:}

Raúl Vallejos Ragas

Jirón Emilio Althaus № 121, Of. 601- Lince. Lima, Perú.

Correo electrónico: raul.vallejos@gmail.com

\section{REFERENCIAS BIBLIOGRÁFICAS}

1. Villena R. Concentración natural de fluoruro en las aguas de consumo de Lima Metropolitana y Callao. [Tesis de Bachiller]. Lima: Universidad Peruana Cayetano Heredia; 1988.

2. Declara la obligación a las empresas procesadoras de sal de consumo a la adición de flúor a este producto. Decreto Supremo No 015-84-SA (Jun. 01, 1984).

3. Aprueba las normas de adición del flúor a la sal de consumo humano. Resolución Ministerial No 0131-85SA/DVM. (May. 31, 1985).

4. Pan American Health Organization. Promoting Oral Health. The Use of Salt Fluoridation to Prevent Dental Caries. Washington (DC): PAHO; 2005.

5. Convenio de Cooperación entre el Ministerio de Salud y Química del Pacifico S.A. "Quimpac". 25 de Julio 1990.

6. Convenio de Cooperación entre el Ministerio de Salud y la Empresa de la Sal S.A. (EMSAL). 28 de Diciembre de 1989.

7. Ministerio de Salud - Organización Panamericana de la Salud. Estudio Epidemiológico de Salud Bucal en el Perú (Caries Dental) 1990. Lima - Perú 1991.

8. Ministerio de Salud. Programas Nacionales. Edición $\mathrm{N}^{\circ}$ 38. May 1992. Especial Salud Pública.

9. Solsona F. Guidelines for Drinking Water Quality Standars in Developing Countries. Regional Advisor in Water Quality. PAHO/CEPIS. Pan American Center for Sanitary Engineering and Environmental Sciences. Division of Health and Environment. Pan American Health Organization. Pan American Sanitary Bureau, 
Regional Office of the World Health Organization. Lima, 2002.

10. Consulta de Registros Sanitarios de Alimentos. Dirección General de Salud Ambiental - DIGESA. URL disponible en: http://www.digesa.sld.pe/ Expedientes/Consulta_Registro_Sanitario.aspx (Fecha de acceso: 5 Mayo 2013).

11. Consulta de RUC. SUNAT. URL disponible en: http:// www.sunat.gob.pe/cl-ti-itmrconsruc/jcrS00Alias (Fecha de acceso: 5 Mayo 2013).

12. De Almeida M. Determinación del número de empresas productoras y comercializadoras, nacionales y extranjeras, de la sal y la disponibilidad de sal con flúor. Informe Final de Consultoría. OPS. 2008. Informe PE/ CNT/0800051.001.

13. Vallejos R. Desafíos de la Salud Pública Bucal en el Perú - análisis crítico. Rev Estomatol Herediana. 2009; 19(1):66-9.

14. Székely M, Bánóczy J, Rugg-Gunn A. A review of worldwide milk fluoridation programs used in caries Prevention. OHDMBSC - Vol. VI - No. 4 - December, 2007.

15. Treasure E, Brown R, Arana A, Chadwick B. 0081 Peruvian Milk Fluoridation Project: cross sectional caries results. URL disponible en: https://iadr.confex. com/iadr/pef06/techprogram/abstract_84642.htm (Fecha de acceso: 3 Junio 2013).

16. Manual de Normas y Procedimientos Técnicos en Odontología de Salud Pública. División de Odontología - Dirección Nacional de Salud - Ministerio de Salud. Lima - 1964.

17. Plan de actividades del Programa de Salud Bucal. Programa de Atención Integral de Salud (PAIS). Ministerio de Salud. 1985.

18. Plan Nacional de Salud Bucal 2005. Resolución Ministerial No 538-2005/MINSA.

19. Vallejos R. Diseño e Implementación del Plan de Atención Integral en el área de Salud Bucal en el ámbito de las direcciones de Salud. Informe final de consultoría. DGSP - MINSA 2004. Contrato No 1343-2004 MINSA.
20. Norma Técnica Sanitaria para la adición de Fluoruros en Cremas Dentales, Enjuagatorios y otros productos utilizados en la Higiene Bucal. Resolución Ministerial No 454 - 2001 SA/DM. (2001).

21. Convenio Interinstitucional entre el Colegio Odontológico del Perú y la empresa Colgate Palmolive Perú. S.A. Lima. Noviembre de 1995.

22. Colegio Odontológico del Perú. Análisis del convenio entre el Colegio Odontológico del Perú y la empresa Colgate Palmolive del Perú. Informe de los miembros de la Comisión de Salud Bucal del COP. Lima. 18 Octubre del 2005.

23. Convenio Marco Interinstitucional entre el Colegio Odontológico del Perú y el Gobierno Regional de Junín. 2013. Resolución Ejecutiva Regional N 426 2013 -GR- JUNIN/PR.

24. Descriptores de Ciencias de la Salud - DeCS. URL disponible en: http://decs.bvs.br/cgi-bin/wxis1660.exe/ decsserver/ (Fecha de acceso: 5 Mayo 2013).

25. Febrer I, Carretero L, et al. Cómo entender un análisis de coste-efectividad. Piel. 2005; 20(4):172-6.

26. Reynolds J, Gaspari K. Operations research methods: Cost-effectiveness. Primary Health Care Operations Research - PRICOR. Agencia de Estados Unidos para el Desarrollo - AID. 1986.

27.C.D.C. Recommendations for Using Fluoride to Prevent and Control Dental Caries in the United States. August 17, 2001/50(RR14); 1-42.

28. Bánóczy J, Petersen PE, Rugg-Gunn AJ (Editors). Milk fluoridation for the prevention of dental caries. World Health Organization, 2009.

29. QuiñonezRB, Stearns SC, TalekarBS, RozierRG, Downs SM. Simulating cost- effectiveness of fluoride varnish during well-child visits for Medicaid-enrolled children. Arch Pediatr Adolesc Med. 2006; 160(2):164-70.

Recibido: 24/09/2014

Aceptado: 15/12/2014 